\title{
Enhancing Lithium Insertion with Electrostatic Nanoconfinement in a Lithography Patterned Precision Cell
}

Sylvia Xin $\mathrm{Li}^{1,2 *}$, Nam S Kim ${ }^{3}$, Kim McKelvey ${ }^{4,5}$, Chanyuan Liu ${ }^{6,7}$, Henry S. White ${ }^{5}$, Gary W. Rubloff ${ }^{6,7}$, Sang Bok Lee ${ }^{3,6}$, Mark A. Reed ${ }^{8 *}$

${ }^{1}$ Department of Physics, Yale University, New Haven, Connecticut 06511, USA,

${ }^{2}$ Department of Chemical Engineering, Massachusetts Institute of Technology, Cambridge, Massachusetts 02139, USA,

${ }^{3}$ Department of Chemistry and Biochemistry, University of Maryland, College Park, Maryland 20742, USA,

${ }^{4}$ Department of Chemistry, University of Utah, Salt Lake City, Utah 84112, USA,

${ }^{5}$ Department of Chemistry, Trinity College Dublin, The University of Dublin, Dublin, D02 PN40, Ireland,

${ }^{6}$ Department of Materials Science and Engineering, University of Maryland, College

Park, Maryland 20742, USA,

${ }^{7}$ Institute for Systems Research, University of Maryland, College Park, Maryland 20742, USA,

${ }^{8}$ Department of Electrical Engineering, Yale University, New Haven, Connecticut 06511, USA

*xinli18@mit.edu

*mark.reed@yale.edu

\section{Supporting Information}

Content Outline:

Supplementary Fig. 1: Nanofabrication flow and cell assembly.

Supplementary Fig. 2: Detailed nanofluidic cell schematic.

Supplementary Fig. 3: Nanofabricated cell characterization.

Supplementary Fig. 4: Redox peak separation of 1 mM LiClO4.

Supplementary Fig. 5: Finite element simulation geometry and boundary conditions.

Supplementary Fig. 6: Conductivity fitted with fitting of native surface charge.

Supplementary Fig. 7: Potential distribution in the electrolyte. 


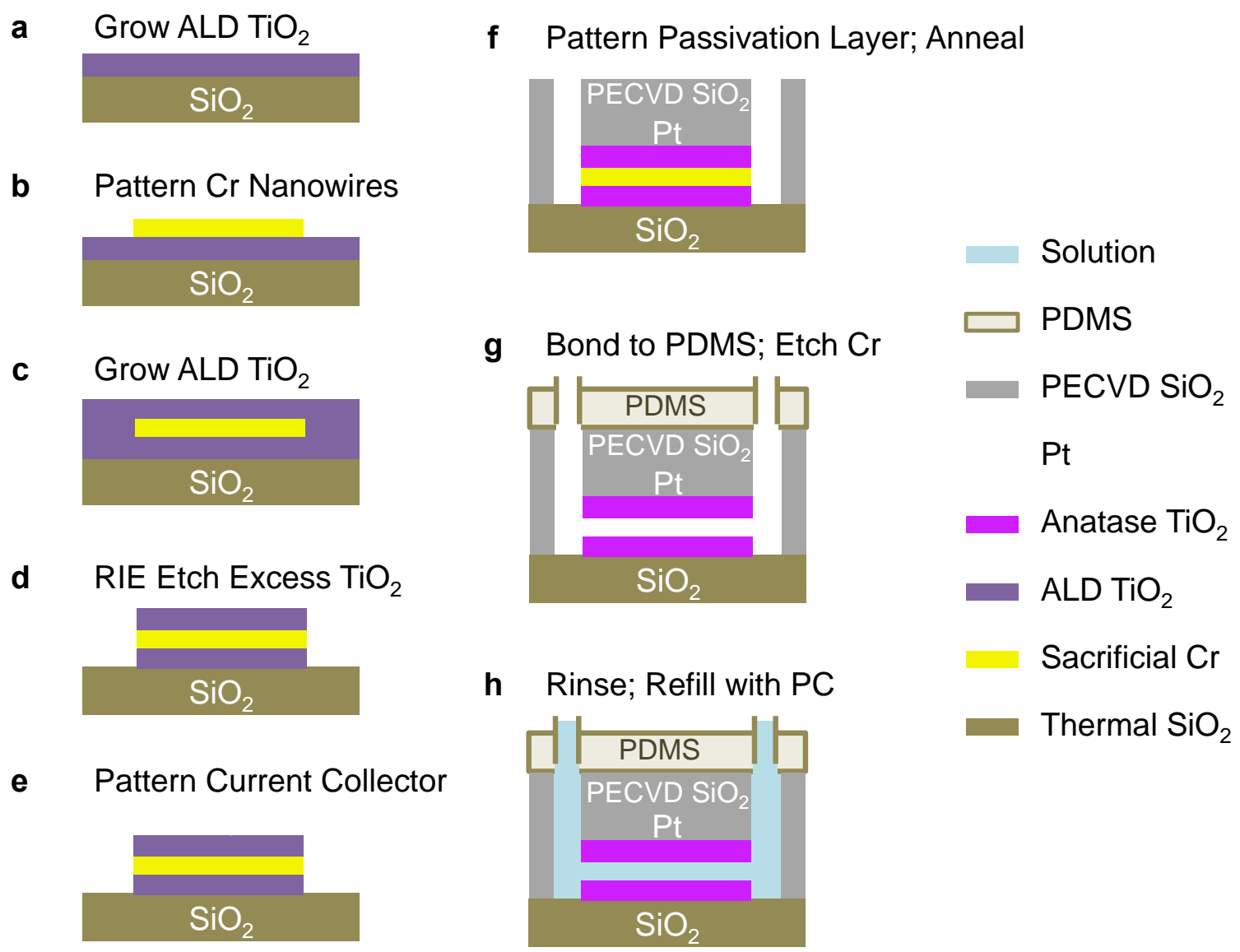

Supplementary Figure 1: Nanofabrication flow and cell assembly. 

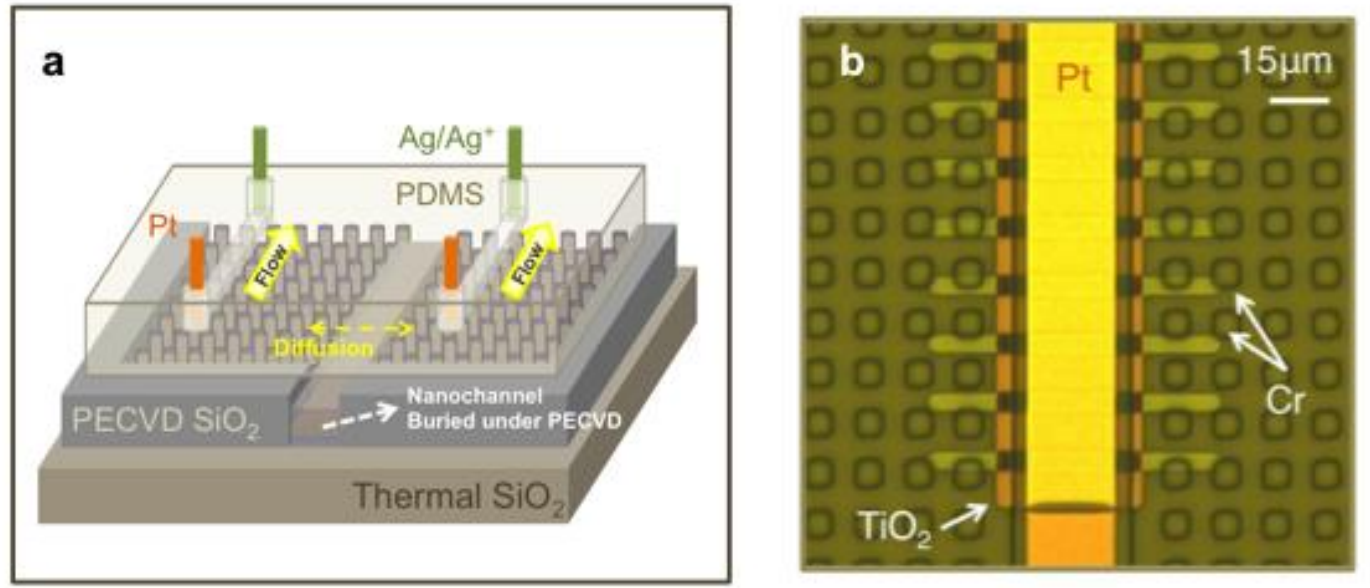

Supplementary Figure 2: Detailed nanofluidic cell schematic. a, Schematic of assembled nanofluidic cell. b, Top-view optical micrograph. 

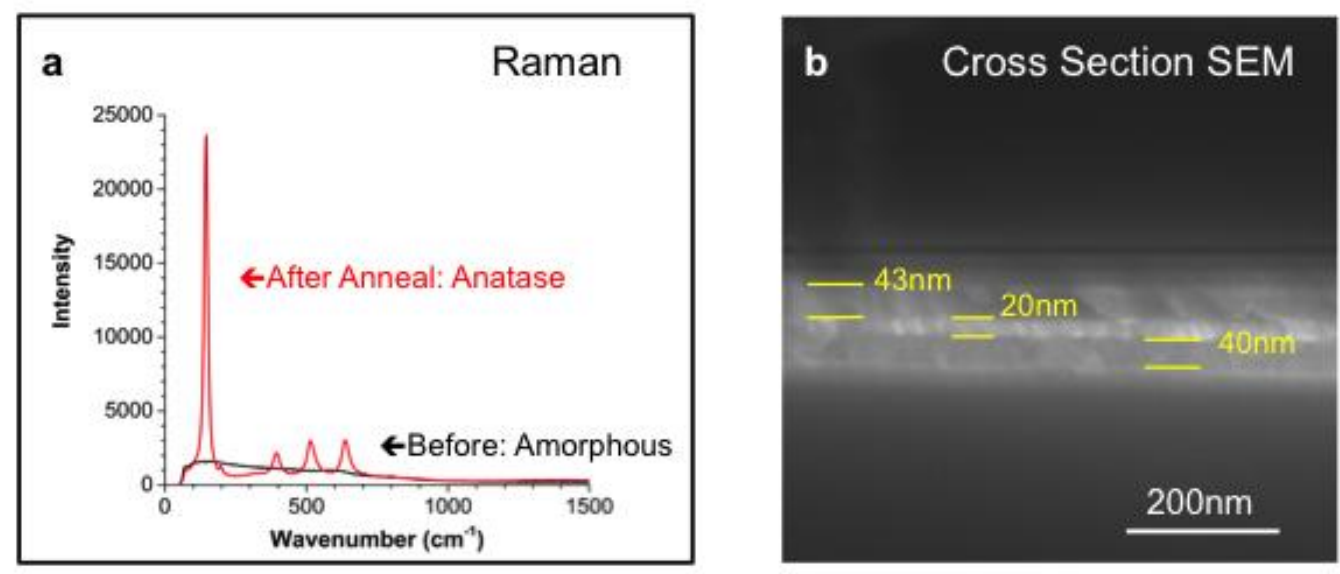

Supplementary Figure 3: Nanofabricated cell characterization. a, Raman spectroscopy of $\mathrm{TiO}_{2}$ before and after annealing. b, SEM cross-section. 


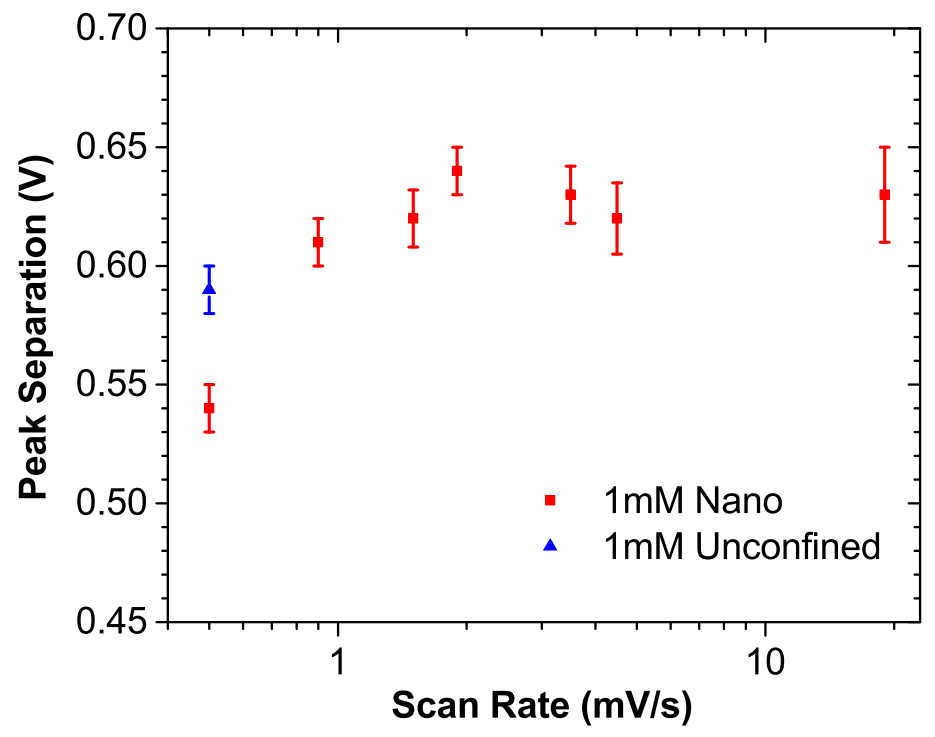

Supplementary Figure 4: Redox peak separation of $1 \mathrm{mM} \mathrm{LiClO}$. For unconfined electrolytes (blue), the peak separation is not observable with scan rate higher than $0.5 \mathrm{mV} / \mathrm{s}$ due to the disappearance of insertion peaks. This is due to potential loss in the electrolyte. In contrast, the peak separation of nanoconfined electrolytes (red) is observable at all tested scan rates. 
a

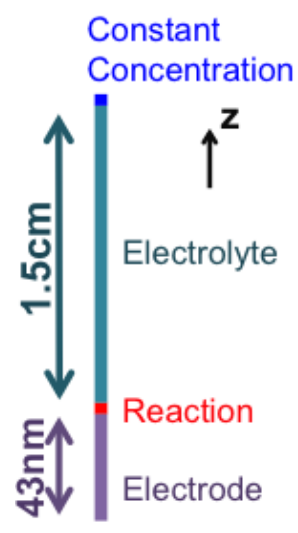

b

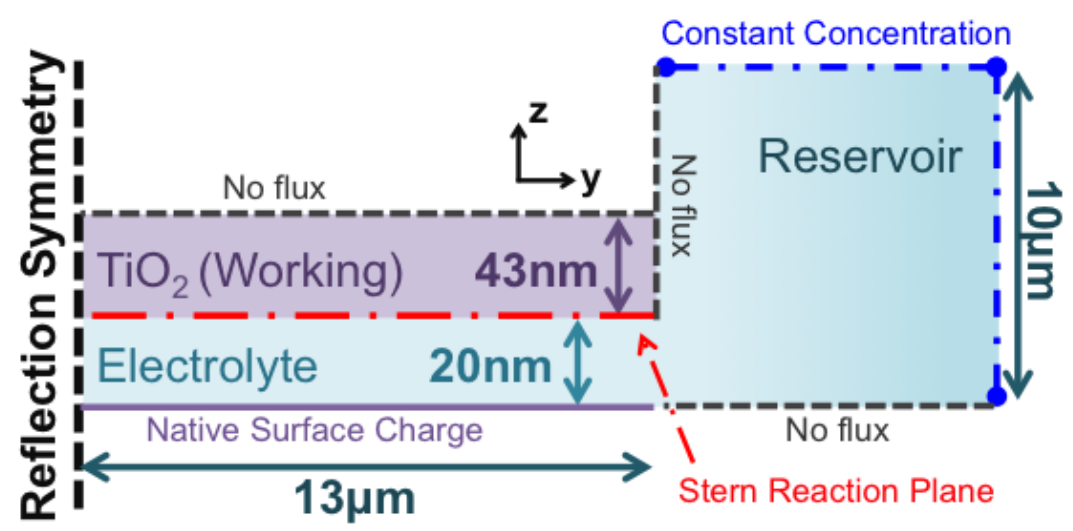

Supplementary Figure 5: Finite element simulation geometry and boundary conditions. a, Unconfined electrolytes, $1 \mathrm{D}$ in z-axis, $\mathrm{TiO}_{2}$ electrode length $43 \mathrm{~nm}$, constant concentration at $1.5 \mathrm{~cm}$ from the reaction position. $\mathbf{b}$, Nanoconfined electrolytes, 2D in yz-plane based on a cross-section of the nanochannel. Boundaries are noted in the figure. At the constant concentration boundaries, $\mathrm{Li}^{+}$and $\mathrm{ClO}_{4}{ }^{-}$concentrations are set to the reservoir values and the electrolyte solution potential $(\phi)$ is set to zero. At the no flux boundaries, the flux of $\mathrm{Li}^{+}$and $\mathrm{ClO}_{4}{ }^{-}$is set to zero. The bottom of the nanochannel also consists of $\mathrm{TiO}_{2}$ with native surface charge $-50 \mathrm{mC} / \mathrm{m}^{2}$, but without a back contact and therefore unconnected in the circuit. Simulating the conductivity along a nanochannel and comparing it to the experimental data, the native surface charge is obtained from the best fitting, which is shown in Supplementary Fig. 6. 


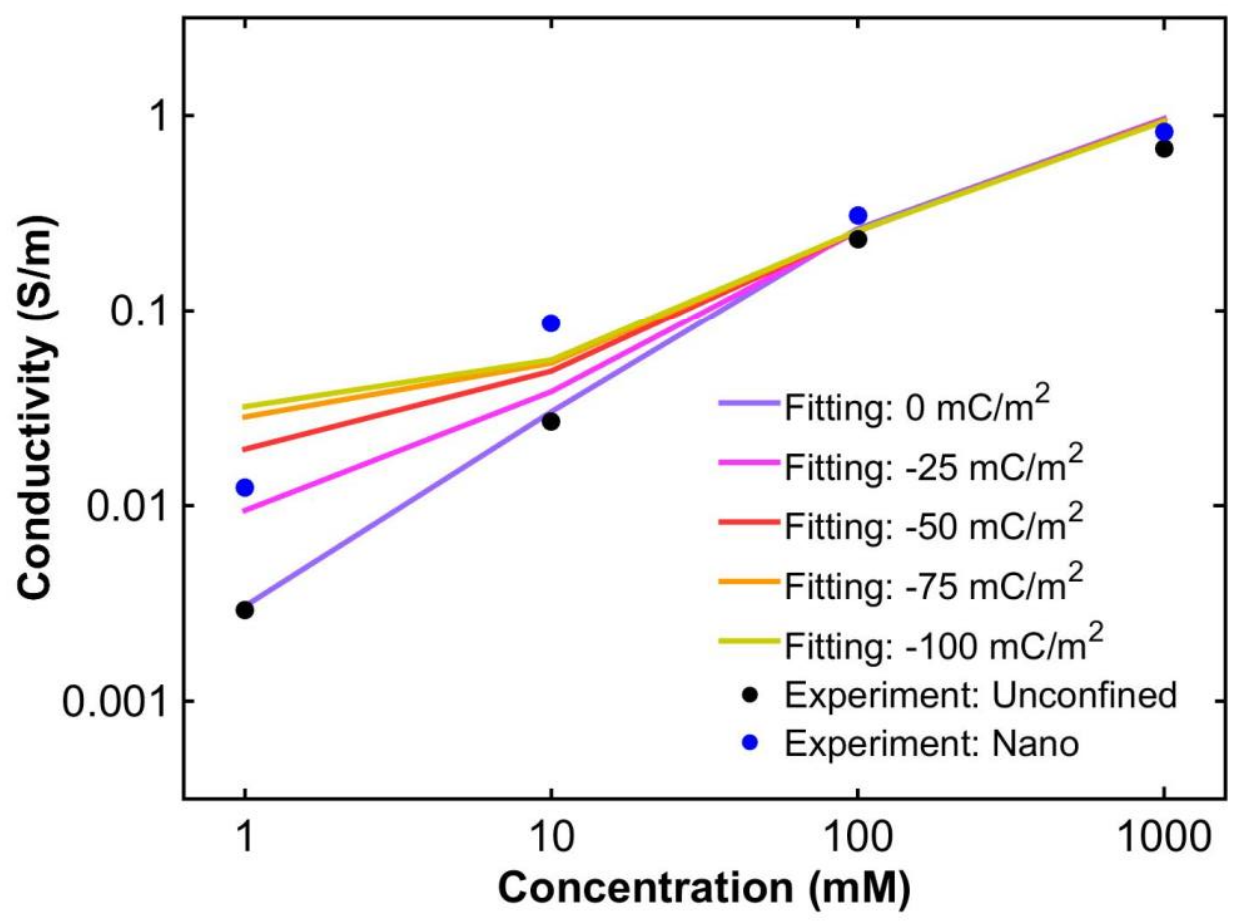

Supplementary Figure 6: Effective conductivity with fitting of simulated native surface charge. Black dots are experimental conductivity of $\mathrm{LiClO}_{4} / \mathrm{PC}$, which is measured in unconfined electrolytes from $1 \mathrm{mM}$ to $1000 \mathrm{mM}$ concentrations by a commercial conductivity meter. Blue dots are experimental data in nanofluidic cells, where two electrodes inserted in the two connecting reservoirs are utilized to measure the conductivity along a nanochannel. The colored lines are fitting results from numerical simulation, with different estimations of native surface charge. $-50 \mathrm{mC} / \mathrm{m}^{2}$ (red line) is decided to be the best fit, and is used to define the bottom wall of a nanochannel in the cyclic voltammetry numerical simulation. 


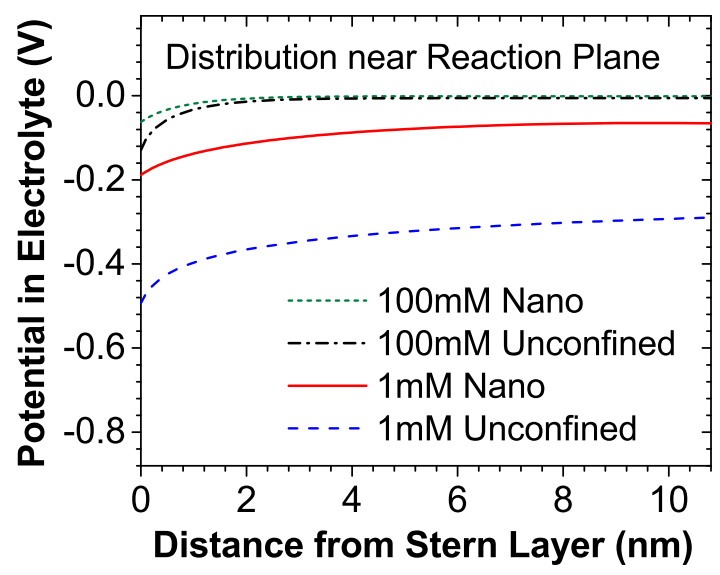

Supplementary Figure 7: Potential distribution in the electrolyte. Simulation result at a snapshot of $-2.45 \mathrm{~V}$, from time dependent numerical simulation with scan rate of $18 \mathrm{mV} / \mathrm{s}$. 